\title{
New hard X-rays and optical polarimetric observations of the Crab nebula and pulsar
}

\author{
Christian Gouiffès* \\ CEA/DRF/IRFU/Service d'Astrophysique \& AIM UMR 7158, France \\ E-mail: christian.gouiffesecea.fr \\ Philippe Laurent \\ CEA/DRF/IRFU/Service d'Astrophysique \& APC UMR 7164 Paris, France \\ E-mail: philippe.laurentecea.fr
}

\section{Andy Shearer}

School of Physics/Centre for Astronomy, NUI Galway, Ireland

E-mail: Andy. shearer@nuigalway.ie

\section{Eoin O'Connor}

School of Physics/Centre for Astronomy, NUI Galway, Ireland

E-mail: eoin1.oconnor@gmail.com

\section{Paul Moran}

School of Physics/Centre for Astronomy, NUI Galway, Ireland

E-mail: paulmoran21@gmail.com

Polarisation is a powerful tool to study compact objects where high-energy particle acceleration processes and magnetic fields play a major role. Joining optical and high-energy polarisation data, whilst challenging, should develop a better understanding of the source acceleration processes and magnetic fields. A recent study of the polarisation from the Crab nebula and pulsar in hard Xray with the International Gamma-Ray Astrophysics Laboratory satellite (INTEGRAL) and in optical with the Galway Astronomical Stokes Polarimeter (GASP) indicated similar variations in the phase average polarisation signal observed between 2005 and 2012. This result was tentatively proposed to evoke that magnetic reconnection was at work in the inner part of the Crab nebula, a physical process suggested to explain the strong flaring activities observed in the past years with the high energy gamma-ray missions Agile and Fermi. We describe here new and higher quality GASP optical data obtained in December 2015 at the WHT telescope at La Palma during nearly simultaneous hard X-rays INTEGRAL/IBIS observations. Our result points to a polarisation angle value higher than that perviously measured, confirming that the inner core of the Crab nebula is variable and cannot be considered as a polarimetric standard.

11th INTEGRAL Conference Gamma-Ray Astrophysics in Multi-Wavelength Perspective 10-14 October 2016

Amsterdam, The Netherlands

\footnotetext{
* Speaker.
} 


\section{Introduction}

The Crab pulsar, remnant of a supernova which occurred in 1054, is a young pulsar powering its surrounding nebula. The Crab pulsar and nebula radiates at all electromagnetic frequencies from the radio to $\mathrm{TeV} \gamma$-rays (1) and as such has been intensively studied with ground and space observatories. Considered for decades as a standard candle in the high energy band, the detection with the Agile and Fermi $\gamma$-ray telescopes from 2007 of strong $\gamma$-ray flares $(2 ; 3 ; 4)$ whose origin is rather unknown have changed this situation and revived the interest and studies of the Crab pulsar/nebula complex. X-ray, Optical and Radio observations did not show any significant flux variation across the nebula (5) during these flaring events and no change of the pulsar's temporal properties was observed. A change in the polarised optical and hard X-rays/ $\gamma$-ray emission of the Crab nebula/pulsar was recently mentioned thanks to a joint study in Optical with the GASP polarimeter and in hard $\mathrm{X}$-rays with the INTEGRAL/IBIS telescope (6). The similar change observed possibly indicates a common origin and triggered new observing campaigns. The present work focus on the description of an optical run conducted in December 2015 at the WHT telescope equipped with the GASP instrument and on data obtained the same year with the INTEGRAL satellite. Throughout this paper the term polarisation refers to phase averaged linear polarisation unless otherwise explicitly specified.

\section{Optical observations}

The optical observations presented here were carried out using the Galway Astronomical Stokes Polarimeter (GASP) at the William Herschel Telescope (WHT) in December, 2015. GASP (7) is a high time resolution, full Stokes, astronomical imaging polarimeter. It currently operates using two Andor iXon 897 Ultra EMCCDs as detectors and operates using the principle of Division of Amplitude Polarimetry (DOAP). The design of GASP is based upon a DOAP concept by Azzam (8) and then later by Compain \& Drevillon (9).

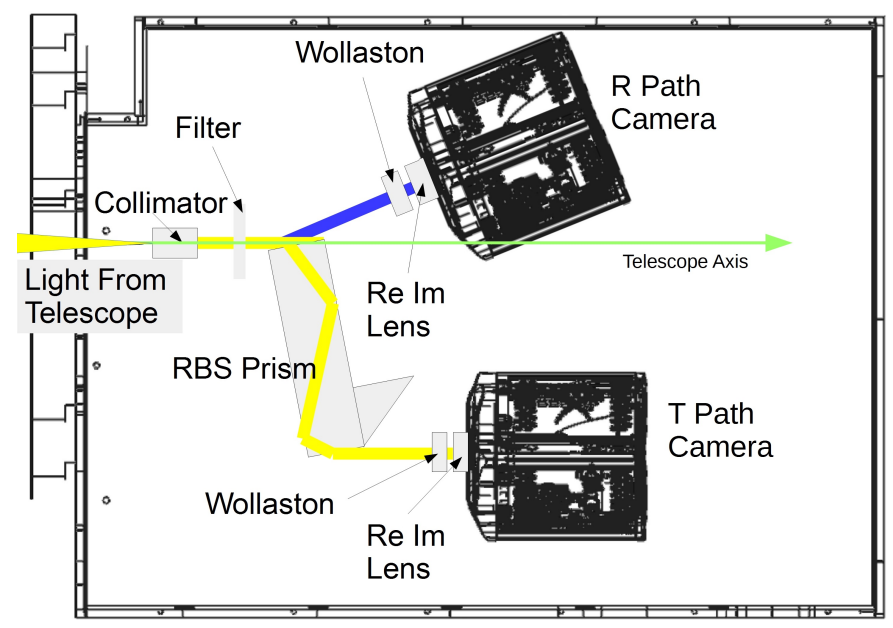

Figure 1: GASP 2D Drawing - showing the layout of the major system components 
GASP utilises a modified Fresnel rhomb (RBS Prism Fig.1), which acts as a highly-achromatic quarter wave plate and a beamsplitter, $36 \%$ of the light (blue path) is split at the first surface of the prism and is re-imaged at the $\mathrm{R}$ Path Camera. The remaining light then undergoes two internal reflections in the prism to add a quarter wave of phase, the circular component is converted to linear which can be examined, this portion of the beam also contains $36 \%$ of the incoming light. Two Wollaston prisms are used to split each of the two optical beams into their orthogonal components and these beams are then re-imaged on the two detectors, resulting in two 25" diameter image fields on each detector. Shown below, Fig. 2., is the optical system presently used in the GASP system.

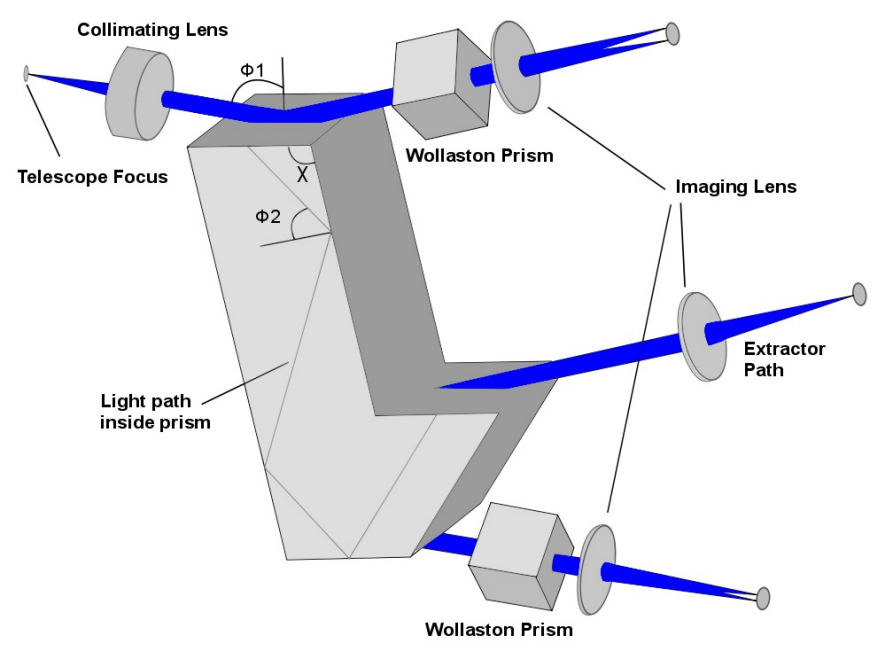

Figure 2: GASP system optical layout.

Two sets of observations were carried out on the Crab pulsar and nebula; the first set consisted of $30 \times 10$ second exposures (starting at MJD57358.08611) with seeing and weather conditions favourable. For this dataset the EMCCD cameras were operated in Full Frame mode (512 x 512 pixels). For the second set of observations (starting at MJD57358.92639) the cameras were operated on a $1.7 \mathrm{~ms}$ cadence for over 2 hours of continuous observation, for this set the EMCCD cameras used a Region of Interest (ROI) to read out a 4x4 binned, $8 \times 128$ pixel region of the sensor. The EM (Electron Multiplying) gain for these observations was set to $\mathrm{x} 300$, resulting in a readout noise of $<0.3 e^{-}$pixel $^{-1}$ using the $17 \mathrm{MHz}$ readout.

Further details of the observations are shown below in Table 1.

Table 1: GASP observational dates and conditions

\begin{tabular}{|c|c|c|c|}
\hline Date & Exposures & Filter & Comments \\
\hline 57358.08611 & $30 \times 10 \mathrm{~s}$ & $\mathrm{R}$ & Seeing was 0.8" for the duration of the exposures \\
\hline $\begin{array}{c}57358.92639- \\
57359.05139\end{array}$ & $6 \times 10^{6} \times 1.7 \mathrm{~ms}$ & $\mathrm{R}$ & $\begin{array}{c}\text { Seeing varied from 0.3" to a maximum of 0.8" } \\
\text { over the duration of the observations }\end{array}$ \\
\hline
\end{tabular}

Table 2 summarises the GASP polarisation standard observations. Due to the plate scale of GASP $(0.14 " /$ pixel), it is not possible to spatially resolve both the pulsar and knot. The fluxes for the four optical paths were extracted using 1.3" radius apertures, which were then annulus sub- 
tracted for each channel. The background-subtracted fluxes from the four channels $I_{1}-I_{4}$ are then converted to a vector and multiplied by the system characterisation matrix (A). The difference in EM gain and variance over the set of images is calibrated out of the system matrix after observations by characterising the EM gain over the sequence of frames. The position angle (P.A.) was referenced to two polarisation standards HD251204 and HD215806. The polarisation measurements were within good agreement with previous observations. A zero polarisation reference star HD 14069 was used to calibrate any residual instrument or telescope induced polarisation.

Table 2: GASP Polarisation Standards (MJD 57358)

\begin{tabular}{|c|c|c|c|c|c|c|}
\hline $\begin{array}{l}\text { Approximate } \\
\text { Polarisation }\end{array}$ & Target & GASP (WHT) & Filter & $\begin{array}{l}\text { Previous Observations } \\
(1990 \text { - 2015) }\end{array}$ & Filter & Ref \\
\hline $0 \%$ & HD 14069 & $0.098_{-0.098}^{+0.187 \%}$ & $\mathrm{R}$ & $0.111 \pm 0.036 \%$ & B & (10) \\
\hline $2 \%$ & HD 215806 & $2.36 \pm 0.12 \%$ & $\mathrm{R}$ & $\begin{array}{l}1.830 \pm 0.040 \% \\
1.3108 \pm 0.1254 \%\end{array}$ & $\begin{array}{l}\mathrm{R} \\
\mathrm{R}\end{array}$ & $\begin{array}{l}\text { (11) } \\
\text { (12) }\end{array}$ \\
\hline $4 \%$ & HD 251204 & $3.98 \pm 0.2 \%$ & $\mathrm{R}$ & $4.04 \pm 0.066 \%$ & $\mathrm{~V}$ & (13) \\
\hline
\end{tabular}

Table 3 shows GASP polarisation measurements of the Crab pulsar. The measurements of the Degree of Linear Polarisation (DOLP) measurements are in very close agreement with previous measurements performed by GASP in 2012 (6) and other instruments including OPTIMA (Optical Pulsar TIMing Analyzer) in 2003 (14). The Position Angle (PA) has been shown to change from measurements performed in 2003 and 2012 and is discussed in more detail in the results section.

Table 3: GASP Observations (WHT December, 2015)

\begin{tabular}{|l|l|l|l|}
\hline Target & Observation Date & Position Angle $\left(^{\circ}\right)$ & Polarisation (\%) \\
\hline Crab Pulsar + Knot & 57358.08611 & $128.42 \pm 0.11$ & $9.77 \pm 0.41$ \\
\hline Crab Pulsar + Knot & 57358.92639 & $130.04 \pm 0.35$ & $9.66 \pm 0.34$ \\
\hline
\end{tabular}

\section{INTEGRAL observations}

Polarimetric monitoring of the Crab pulsar and nebula in the hard X-rays/low- $\gamma$-rays band with the IBIS telescope on board INTEGRAL was possible since launch thanks to its use as a Compton telescope. Briefly, The polarisation of celestial sources is measured using Compton scattering properties. Photons entering IBIS are Compton scattered in the first detector plane, the INTEGRAL Soft Gamma-Ray Imager (ISGRI), and then absorbed in the second detector, the Pixelated Cesium Iodide Telescope detector (PICsIT). Use in this mode, IBIS is a coded-aperture Compton telescope that provides high-energy response, low background, and a wide FOV see Forot et al, 2007 (15) and references therein for methods and details. The Crab nebula is regularly observed since launch with INTEGRAL for calibration purposes and polarisation results obtained with IBIS until 2014 were published (16; 6). For this work we used data obtained between 2015, August 18 and October 17 for a total exposure time of 500ksec, a period close to our WHT optical observations. To improve the 
quality of the extracted signal, we use a new method that optimises the selection of the Compton events in the data analysis process. In particular, adjusting carefully a parameter (the rise-time value) of ISGRI events, has shown a decrease in the proportion of spurious events, thus improving the signal (see Laurent et al in these proceedings to a description of this original method). The result of this analysis is shown in Figure 3.

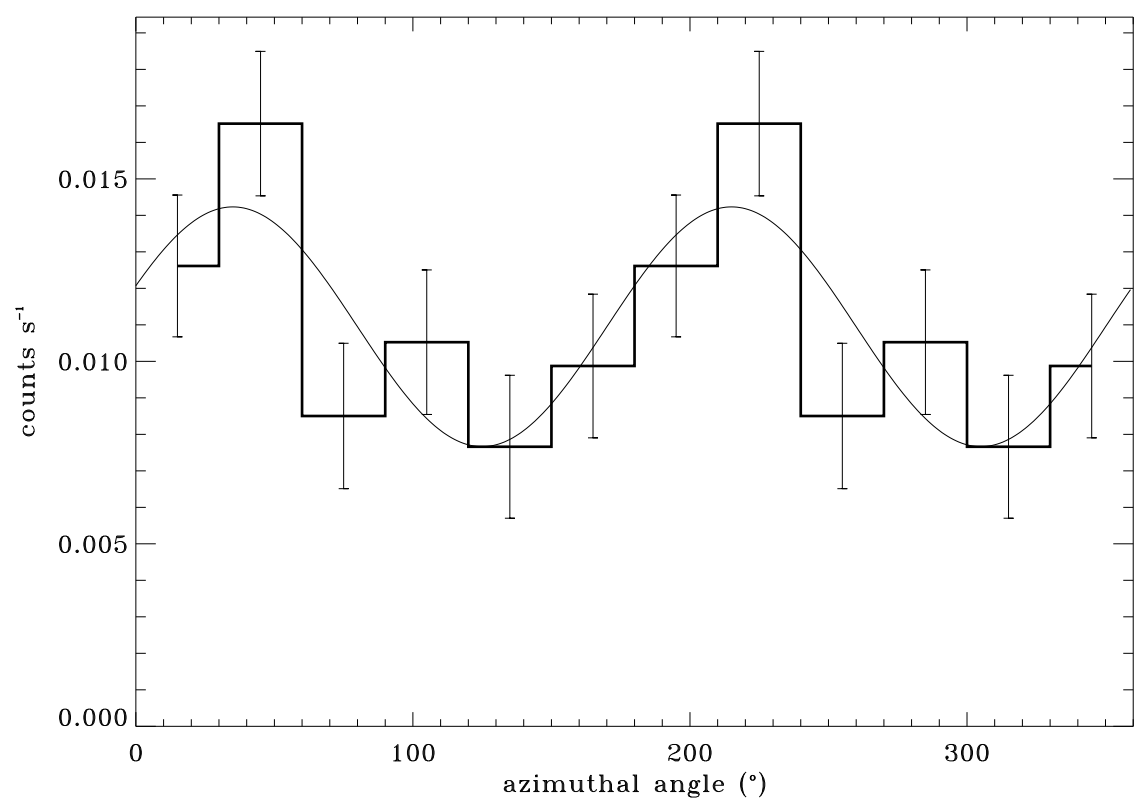

Figure 3: Phase-averaged polarisation diagram of the Crab pulsar/nebula obtained by INTEGRAL/IBIS in fall 2015 in the 300-450 keV energy band. The distribution or polarigramme gives the source count rate by azimuthal angle of the Compton scattering. The minimum of the sinusoidal fit indicates a polarisation angle of $125^{\circ} \pm 15^{\circ}$, a polarisation fraction of $89 \pm 28$ and a $\mathrm{S} / \mathrm{N}$ ratio of 14.

The parameters measured at different epochs with INTEGRAL/IBIS give the indication, even with a relatively modest significance, of a change of the polarisation angle of the Crab pulsar/nebula, see Table 4.

Table 4: INTEGRAL/IBIS polarisation parameters in the [300-450] keV band

\begin{tabular}{|l|l|l|l|}
\hline Observation Date & Position Angle $\left(^{\circ}\right)$ & Polarisation fraction & Reference \\
\hline $2003-2007$ & $115 \pm 11$ & $96 \pm 34$ & $(16)$ \\
\hline $2012-2014$ & $80 \pm 12$ & $98 \pm 37$ & $(6)$ \\
\hline 2015 & $125 \pm 15$ & $89 \pm 28$ & This work \\
\hline
\end{tabular}

\section{Discussion and Conclusion}

Our combined 2015 observing campaign indicates that in both optical and hard X-rays a polarisation angle with a value higher than previously measured (see Figure 4). The statistical significance 
of the INTEGRAL point is modest (estimated to have an equivalent $3 \sigma$ detection level (see (6) for a discussion on the INTEGRAL polarigram errors), but consistent with the value derived from the WHT+GASP observation. It is worthwhile to mention that concerning the INTEGRAL/IBIS polarisation angle value, data taken by the ASTROSAT mission at the same epoch and in a similar energy range similar, is consistent with our result (D. Bhattacharya, this conference).

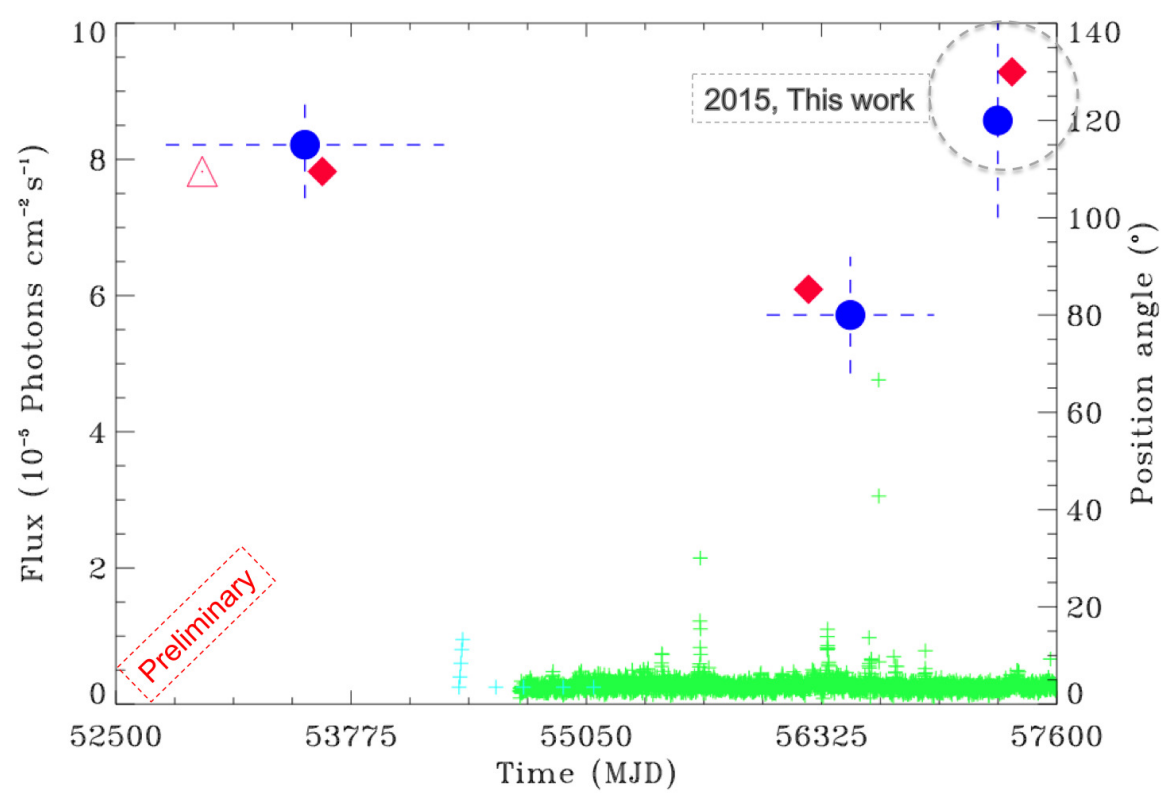

Figure 4: Position angle of the Crab pulsar/nebula versus time measured in Optical and in Hard X-rays. Optical: - in red open triangle: OPTIMA, 2003 (14), - in red filled diamond: HST in 2005, GASP at Palomar 200 inch in 2012 (14) and GASP at WHT in December 2015 (this work, inside the dashed grey line circle). Hard X-rays : INTEGRAL results (in blue filled circle) obtained in the period 2003-2007 (16), 2012-2014 (14) and in 2015 (this work). The axis on the right side is expressed in degrees. The activity of the source observed during the past years at energy greater than $100 \mathrm{MeV}$ is plotted with cyan (AGILE satellite) and green (FERMI observatory) colours .

At this stage, It is tempting to attribute a common origin to these similar optical and hard Xrays polarisation changes and magnetic reconnection with a change in the overall magnetic field orientation is suggested. Changes in the optical polarisation from AGN have been observed and associated with $\gamma$-ray flares (17) and the variations detected inside the inner core of the Crab nebula might be a smaller case of such phenomenon. Clearly, more date are required and we will continue to monitor this source. In particular, we are currently investigating the phase resolved polarisation signal, in optical and with INTEGRAL, thanks to the excellent timing resolution of both instruments. This will allow to better discriminate, especially at high energy where spatial resolution cannot compete with the sharpness of the optical images, what is coming from the pulsar and what from the inner knot.

Optical, Hard X-rays and $\gamma$-ray polarisation studies are a powerful tool to investigate the geometry and magnetic configuration of many class of sources. In that respect, the INTEGRAL results 
are encouraging and might pave the way to future researches, especially since the recently achieved technical progresses. The coming and/or proposed space missions like IXPE, XIPE, EXTP, eASTROGAM and many others are promising and will surely open new opportunities. On the optical and near-infrared side, even if the domain is more mature, we hope that the instrumental plans for the next generation of giant telescopes and/or the future/up-graded equipments of existing facilities will allow similar progresses and bring into reality very sensitive multi-wavelength time resolved polarimetric studies.

\section{Acknowledgments}

This work is partly based on observations with INTEGRAL, a European Space Agency (ESA) project with instruments and science data center funded by ESA member states (especially the Principal Investigator countries: Denmark, France, Germany, Italy, Switzerland, and Spain), Czech Republic and Poland, and with the participation of Russia and the United States. The authors are grateful for time allocated for GASP observations at WHT La Palma under the Opticon programme. FP7/2007-2013: The research leading to these results has received funding from the European Community's Seventh Framework Programme (FP7/2007-2013) under grant agreement number RG226604 (OPTICON). C.G. and P.L. acknowledge financial support from the French Space Agency (CNES). EOC would like to acknowledge funding from the Irish Research Council under GOIPG/2014/1266. 


\section{References}

[1] J. J. Hester, The Crab Nebula: An Astrophysical Chimera, ARA\&A 46 (Sept., 2008) $127-155$.

[2] A. A. Abdo, M. Ackermann, M. Ajello, A. Allafort, L. Baldini, J. Ballet et al., Gamma-Ray Flares from the Crab Nebula, Science 331 (Feb., 2011) 739-, [1011.3855].

[3] M. Tavani, A. Bulgarelli, V. Vittorini, A. Pellizzoni, E. Striani, P. Caraveo et al., Discovery of Powerful Gamma-Ray Flares from the Crab Nebula, Science 331 (Feb., 2011) 736-, [1101.2311].

[4] E. Striani, M. Tavani, V. Vittorini, I. Donnarumma, A. Giuliani, G. Pucella et al., Variable Gamma-Ray Emission from the Crab Nebula: Short Flares and Long "Waves", ApJ 765 (Mar., 2013) 52, [1302.4342].

[5] M. C. Weisskopf, A. F. Tennant, J. Arons, R. Blandford, R. Buehler, P. Caraveo et al., Chandra, Keck, and VLA Observations of the Crab Nebula during the 2011-April Gamma-Ray Flare, ApJ 765 (Mar., 2013) 56, [1211.3997].

[6] P. Moran, G. Kyne, C. Gouiffès, P. Laurent, G. Hallinan, R. M. Redfern et al., A recent change in the optical and $\gamma$-ray polarization of the Crab nebula and pulsar, MNRAS 456 (Mar., 2016) 2974-2981, [1511.07641].

[7] P. Collins, G. Kyne, D. Lara, M. Redfern, A. Shearer and B. Sheehan, The Galway astronomical Stokes polarimeter: an all-Stokes optical polarimeter with ultra-high time resolution, Experimental Astronomy 36 (Dec., 2013) 479-503, [1305. 6825].

[8] R. Azzam, Division-of-amplitude photopolarimeter (doap) for the simultaneous measurement of all four stokes parameters of light, Optica Acta: International Journal of Optics 29 (1982) 685-689, [http://dx.doi.org/10.1080/713820903].

[9] E. Compain, S. Poirier and B. Drevillon, General and self-consistent method for the calibration of polarization modulators, polarimeters, and mueller-matrix ellipsometers, Appl. Opt. 38 (Jun, 1999) 3490-3502.

[10] G. D. Schmidt, R. Elston and O. L. Lupie, The Hubble Space Telescope Northern-Hemisphere grid of stellar polarimetric standards, AJ 104 (Oct., 1992) 1563-1567.

[11] D. C. B. Whittet, P. G. Martin, J. H. Hough, M. F. Rouse, J. A. Bailey and D. J. Axon, Systematic variations in the wavelength dependence of interstellar linear polarization, ApJ 386 (Feb., 1992) 562-577.

[12] A. Słowikowska, K. Krzeszowski, M. Żejmo, P. Reig and I. Steele, Calibration of the Liverpool Telescope RINGO3 polarimeter, MNRAS 458 (May, 2016) 759-771, [1602.02105]. 
[13] D. A. Turnshek, R. C. Bohlin, R. L. Williamson, II, O. L. Lupie, J. Koornneef and D. H. Morgan, An atlas of Hubble Space Telescope photometric, spectrophotometric, and polarimetric calibration objects, AJ 99 (Apr., 1990) 1243-1261.

[14] A. Słowikowska, G. Kanbach, M. Kramer and A. Stefanescu, Optical polarization of the Crab pulsar: precision measurements and comparison to the radio emission, MNRAS 397 (July, 2009) 103-123, [0901.4559].

[15] M. Forot, P. Laurent, F. Lebrun and O. Limousin, Compton Telescope with a Coded Aperture Mask: Imaging with the INTEGRAL/IBIS Compton Mode, ApJ 668 (Oct., 2007) 1259-1265, [0708.3724].

[16] M. Forot, P. Laurent, I. A. Grenier, C. Gouiffès and F. Lebrun, Polarization of the Crab Pulsar and Nebula as Observed by the INTEGRAL/IBIS Telescope, ApJ 688 (Nov., 2008) L29-L32, [0 809 . 1292].

[17] A. A. Abdo, M. Ackermann, M. Ajello, M. Axelsson, L. Baldini, J. Ballet et al., A change in the optical polarization associated with a $\gamma$-ray flare in the blazar 3C279, Nature 463 (Feb., 2010) 919-923, [1004.3828]. 